\title{
Genome Resources for the Ex-Type of Phytophthora citricola, and Well-Authenticated Isolates of $P$. hibernalis, $P$. nicotianae, and $P$. syringae
}

\author{
Subodh K. Srivastava, ${ }^{1,2, \dagger}$ Leandra M. Knight, ${ }^{1,2}$ Mark Nakhla, ${ }^{1}$ and Z. Gloria Abad ${ }^{1, \dagger}$ \\ ${ }^{1}$ United States Department of Agriculture-Animal and Plant Health Inspection Service Plant Protection \\ and Quarantine, S\&T, Pathogen Confirmatory Diagnostics Laboratory, Beltsville, MD 20705 \\ ${ }^{2}$ Department of Entomology and Plant Pathology, North Carolina State University, Raleigh, NC 27695
}

\section{Funding}

This research was supported by the United States Department of Agriculture-Animal and Plant Health Inspection Service Farm Bill Section 10007 funding (FY 2018, 3.0630.01) provided to G. Abad.

\section{Keywords}

bioinformatics, oomycetes, pathogen detection

\section{Resource Announcement}

Phytophthora is one of the most important genera of plant pathogens, with many members causing high economic and environmental impact diseases in nurseries, forests, and natural ecosystems. To build robust molecular identification systems for effective management of plant-pathogenic species of Phytophthora, it is very important to have information from wellauthenticated specimens and, preferably, the ex-type specimens (Martin et al. 2012). The ex-type materials are living cultures (and duplicates of the type) deposited in international culture collections as a requirement for the official description of new species. The reference genomes from well-authenticated specimens form a critical foundation for genetics, biological research, and diagnostic applications. Until 2019, there were 83 genome assemblies for 22 species of Phytophthora at the NCBI; however, only two genomes were from ex-types. These genomes were for Phytophthora lateralis (Feau et al. 2016) and P. agathidicida) (Studholme et al. 2015). In this article, we describe draft genome resources for four additional Phytophthora spp. acquired from the World Phytophthora Collection (WPC) at the University of California, Riverside that were generated using MinION long-read high-throughput sequencing technology

${ }^{\dagger}$ Corresponding authors: Z. G. Abad; Gloria.Abad@usda.gov, and

S. K. Srivastava; Subodh.k.srivastava@usda.gov

The author(s) declare no conflict of interest.

Accepted for publication 15 October 2021.

(c) 2022 The American Phytopathological Society 
Table 1. Phytophthora ex-type and well-authenticated species generated genome assembly statistics in this study using Nanopore sequencer and Canu assembler ${ }^{\mathrm{a}}$

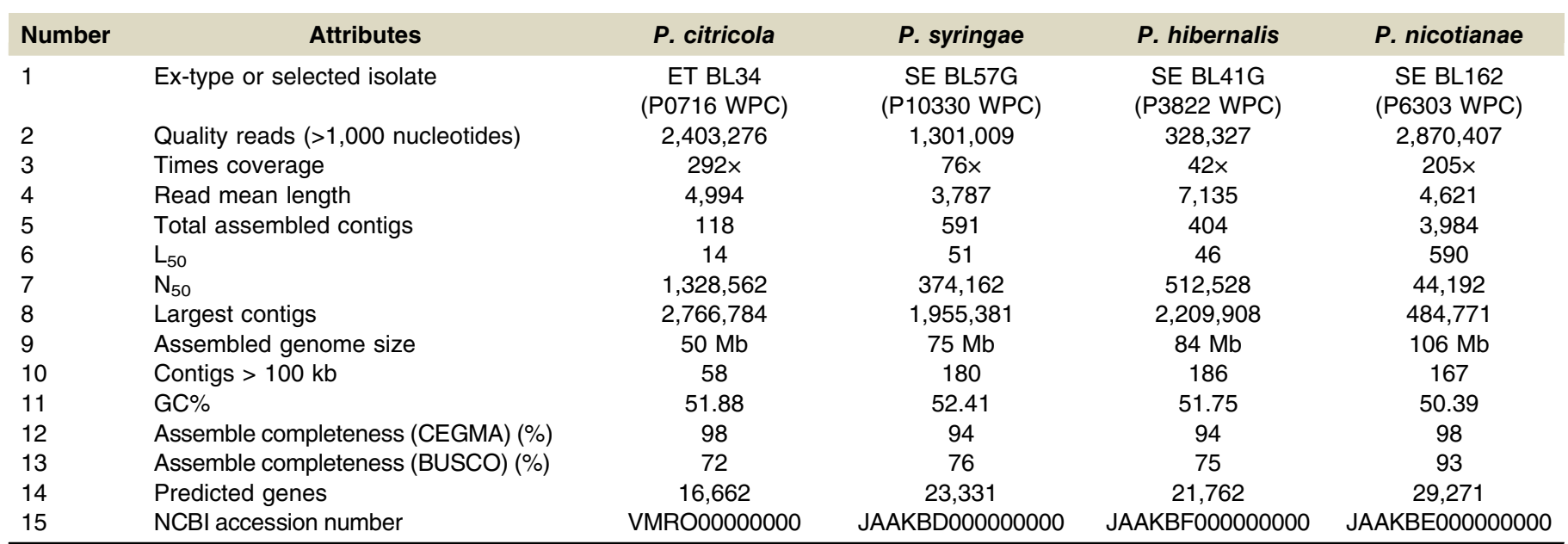

\footnotetext{
${ }^{a}$ Abbreviations: ET = ex-type material; SE = selective specimen; WPC = World Phytophthora Collection, Riverside, CA, U.S.A.; CEGMA = Core Eukaryotic Genes Mapping Approach (core eukaryotic gene set); and BUSCO = Benchmarking Universal Single-Copy Orthologs (single copy gene set).
}

(Oxford Nanopore Technologies [ONT]). These species include the ex-type of $P$. citricola isolate $\mathrm{BL} 34$ (P0716 WPC) as well as the well-authenticated P. hibernalis isolate 41G (P3822 WPC), P. nicotianae isolate BL162 (P6303 WPC), and P. syringae isolate BL57G (P10330 WPC). We selected well-authenticated specimens for $P$. hibernalis, P. nicotianae, and $P$. syringae for species that have lost the ex-types at all international culture collections with information available at the "IDphy" International Online Resource for Phytophthora http://idtools.org/id/ phytophthora/about.php (Abad et al. 2019). High-quality DNA (approximately 100 to $200 \mathrm{ng}$ ) was obtained from pure mycelial cultures using the Qiagen DNeasy Mericon Food Kit protocol for DNA extraction, modified to be used for Phytophthora spp. from the Abdel-Latif and Osman (2017) protocol for corn. The quality of the genomic DNA was determined using Qubit, Nanodrop, and Tape station (Agilent Technologies) and the genomic DNA libraries were prepared from 100 to $200 \mathrm{ng}$ using the ONT ligation sequencing kit SQK-LSK109 (Abdel-Latif and Osman 2017; Srivastava et al. 2020). The raw reads were generated on a flowcell R9.4.1 in a MinlON device for $48 \mathrm{~h}$ using ONT's sequencing protocol, resulting in 2.4 million reads for $P$. citricola, 1.3 million reads for $P$. syringae, 300,000 reads for $P$. hibernalis, and 2.8 million reads for $P$. nicotianae, and quality filtered reads (after filtered low quality and $<7 \mathrm{Q}$-score) were processed for sequence data conversion from Fast5 to FastQ with the guppy basecaller (Wick et al. 2019). We used filtered quality data having median read quality of $10.3,11.6,12.3$, and 12.4 in these four assembled genomes (i.e., $P$. citricola, $P$. hibernalis, $P$. nicotiana, and $P$. syringae respectively). These quality reads were assembled and received high coverage for genomes using Canu assembly (Koren et al. 2017) and the contigs were polished with ONT reads to recorrect any error bases using Pilon (Walker et al. 2014). Finally, we evaluated the assembled contigs with quality assessment tools for genome assemblies known as QUAST (Gurevich et al. 2013) and CEGMA model sets (Nishimura et al. 2017; Simão et al. 2015). We trained the prediction model using a previously sequenced species ( $P$. capsici) as a model organism for AUGUSTUS software (Cui et al. 2019; Stanke and Waack 2003; Stanke et al. 2008; Srivastava et al. 2020) and predicted proteins (Table 1). The analyses of available genomes of $P$. nicotianae genomes at the NCBI were used for comparative analysis with the P. nicotianae isolate BL162 (P6303 WPC) from Gramatophyllum sp. from Indonesia, mating type (A2) within species. The Canu assembly reported 834 repeats of a total length of $11,869197 \mathrm{bp}$ adding to this genome with the ex-type. This assembled genome showed variation with other previously submitted genomes (races) of the size of $P$. nicotianae race 0 and race 1 (Blair et al. 2008; Liu et al. 2016). Therefore, we did comparative genome analysis between sequenced species genic regions and predicted genes using the AUGUSTUS pipeline described previously (Srivastava et al. 2020, 2021), and extended the analysis with other sequenced $P$. nicotianae subspecies (BL162 with 29,260 genes, race 0 with 23,619 genes, race 01 with 20,170 genes, and GM01 with 16,776 genes). The comparative gene analysis 
with sequenced species (BL162) revealed 5,641 additional genes in P. nicotianae BL162, and 318 were unique genes compared with the ex-type. We extended analysis using the NCBI nonredundant database, resulting in 135 unique genes. We analyzed PFAM domain annotations among these genomewide and found significant protein domain numbers for some important species; that is, RXLR phytopathogen effector protein, protein kinase domain, and pectate lyase in $P$. citricola $=111,320$, and 46 domains, respectively; in $P$. hibernalis $=157,304$, and 48 domains; in $P$. nicotianae $=240,370$, and 104 domains, respectively; and in $P$. syringae; 71,336 , and 47 domains, respectively.

In conclusion, we developed draft genome assemblies for four additional Phytophthora spp. from type and well-authenticated isolates for various research and diagnostic applications and have deposited these at the NCBI GenBank databases.

The whole-genome sequences of this project have been deposited into NCBI GenBank project PRJNA555328 for $P$. citricola BL34 (P0716 WPC) accession number VMR000000000 with Sequence Read Archive (SRA) submission SRR9942578; the other three under project PRJNA605765 include $P$. syringae BL57G (P10330 WPC) accession number JAAKBD000000000 with SRA submission SRR11103462, P. nicotianae BL162 (P6303) accession number JAAKBE000000000 with SRA submission SRR11103463, and $P$. hibernalis BL41G (P3822 WPC) accession number JAAKBF000000000 with SRA submission SRR11103464.

\section{Acknowledgments}

We thank Y. Rivera and K. Zeller from the United States Department of Agriculture-Animal and Plant Health Inspection Service Plant Protection and Quarantine S\&T, Plant Pathogen Confirmatory Laboratory (former Beltsville Laboratory) for their support in this project and their suggestions for improvement of the manuscript.

\section{Literature Cited}

Abad, Z. G., Burgess, T., Bienapfl, J. C., Redford, A. J., Coffey, M., and Knight, L. 2019. IDphy: Molecular and morphological identification of Phytophthora species based on the types and other well-authenticated specimens. USDA APHIS PPQ S\&T Beltsville Lab, USDA APHIS PPQ S\&T ITP, Centre for Phytophthora Science and Management, and World Phytophthora Collection. https://idtools.org/id/ phytophthora/index.php

Abdel-Latif, A., and Osman, G. 2017. Comparison of three genomic DNA extraction methods to obtain high DNA quality from maize. Plant Methods 13:1.

Blair, J. E., Coffey, M. D., Park, S. Y., Geiser, D. M., and Kang, S. 2008. A multilocus phylogeny for Phytophthora utilizing markers derived from complete genome sequences. Fungal Genet. Biol. 45:266-277.

Cui, C., Herlihy, J., Bombarely, A., McDowell, J. M., and Haak, D. C. 2019. Draft assembly of Phytophthora capsici from long-read sequencing uncovers complexity. Mol. Plant-Microbe Interact. 32:1559-1563.

Feau, N., Taylor, G., Dale, A. G., Dhillon, B., Bilodeau, G. J., Birol, I., Jones, S. J. M., and Hamelin, R. C. 2016. Genome sequences of six Phytophthora species threatening forest ecosystems. Genome Data 10:85-88.

Gurevich, A., Saveliev, V., Vyahhi, N., and Tesler, G. 2013. QUAST: Quality assessment tool for genome assemblies. Bioinformatics 29:1072-1075.

Koren, S., Walenz, B. P., Berlin, K., Miller, J. R., Bergman, N. H., and Phillippy, A. M. 2017. Canu: Scalable and accurate long-read assembly via adaptive k-mer weighting and repeat separation. Genome Res. 27:722-736.

Liu, H., Ma, X., Yu, H., Fang, D., Li, Y., Wang, X., Wang, W., Dong, Y., and Xiao, B. 2016. Genomes and virulence difference between two physiological races of Phytophthora nicotianae. Gigascience 5:3.

Martin, F. N., Abad, Z. G., Balci, Y., and Ivors, K. 2012. Identification and detection of Phytophthora: Reviewing our progress, identifying our needs. Plant Dis. 96: 1080-1103.
Nishimura, O., Hara, Y., and Kuraku, S. 2017. gVolante for standardizing completeness assessment of genome and transcriptome assemblies. Bioinformatics 33 : 3635-3637.

Simão, F. A., Waterhouse, R. M., loannidis, P., Kriventseva, E. V., and Zdobnov, E. M. 2015. BUSCO: Assessing genome assembly and annotation completeness with single-copy orthologs. Bioinformatics 31:3210-3212.

Srivastava, S. K., Abad, Z. G., Knight, L. M., Zeller, K., Mavrodieva, V., and Nakhla, M. 2020. Draft genome resource for the ex-types of Phytophthora ramorum, $P$. kernoviae, and $P$. melonis, species of regulatory concern, using ultra-long read MinION nanopore sequencing. Mol. Plant-Microbe Interact. 33:794-797.

Srivastava, S. K., Zeller, K. A., Sobieraj, J. H., and Nakhla, M. K. 2021. Genome resources of four distinct pathogenic races within Fusarium oxysporum f. sp. vasinfectum that cause vascular wilt disease of cotton. Phytopathology 111:593596.

Stanke, M., Diekhans, M., Baertsch, R., and Haussler, D. 2008. Using native and syntenically mapped CDNA alignments to improve de novo gene finding. Bioinformatics 24:637-644.

Stanke, M., and Waack, S. 2003. Gene prediction with a hidden Markov model and a new intron submodel. Bioinformatics :ii215-ii225.

Studholme, D. J., McDougal, R. L., Sambles, C., Hansen, E., Hardy, G., Grant, M., Ganley, R. J., and Williams, N. M. 2015. Genome sequences of six Phytophthora species associated with forests in New Zealand. Genome Data 7: 54-56.

Walker, B. J., Abeel, T., Shea, T., Priest, M., Abouelliel, A, Sakthikumar, S., Cuomo, C. A., Zeng, Q., Wortman, J., Young, S. K., and Earl, A. M. 2014. Pilon: An integrated tool for comprehensive microbial variant detection and genome assembly improvement. PLoS One 9:e112963.

Wick, R. R., Judd, L. M., and Holt, K. E. 2019. Performance of neural network basecalling tools for Oxford Nanopore sequencing. Genome Biol. 20:129. 